\title{
Characterization of isoforms of activin receptor-interacting protein 2 that augment activin signaling
}

\author{
Z H Liu, K Tsuchida, T Matsuzaki, Y L Bao, A Kurisaki \\ and $\mathbf{H}$ Sugino
}

The Institute for Enzyme Research, University of Tokushima, 3-18-15 Kuramoto, Tokushima 770-8503, Japan
(Requests for offprints should be addressed to K Tsuchida at Fujita Health University; Email: tsuchida@fujita-hu.ac.jp)
(Z H Liu is now at School of Basic Medical Sciences, Jilin University, 2 Xinmin Street, Changchun 130021, China)
(K Tsuchida is now at Division for Therapies Against Intractable Diseases, Institute for Comprehensive Medical Science, Fujita Health University, Toyoake, Aichi
$\quad 470-1192$, Japan)
(T Matsuzaki is now at Department of Biology, Faculty of Sciences, Kyushu University Graduate School, Fukuoka 812-8581, Japan)
(Y L Bao is now at The Institute of Genetics and Cytology, Northeast Normal University, 5268 Renmin St, Changchun 130024, China)

\begin{abstract}
Activin type II receptors (ActRIIs) including ActRIIA and ActRIIB are serine/threonine kinase receptors that form complexes with type I receptors to transmit intracellular signaling of activins, nodal, myostatin and a subset of bone morphogenetic proteins. ActRIIs are unique among serine/threonine kinase receptors in that they associate with proteins having PSD-95, Discs large and ZO-1 (PDZ) domains. In our previous studies, we reported specific interactions of ActRIIs with two independent PDZ proteins named activin receptor-interacting proteins 1 and 2 (ARIP1 and ARIP2). Overexpression of both ARIP1 and ARIP2 reduce activin-induced transcription. Here, we report the isolation of two isoforms of ARIP2 named ARIP2b and 2c. ARIP2, ARIP2b and ARIP2c recognize COOH-terminal residues of ActRIIA
\end{abstract}

that match a PDZ-binding consensus motif. ARIP2 and its isoforms have one $\mathrm{PDZ}$ domain in the $\mathrm{NH}_{2}$-terminal region, and interact with ActRIIA. Although PDZ domains containing GLGF motifs of ARIP2b and $2 c$ are identical to that of ARIP2, their $\mathrm{COOH}$-terminal sequences differ from that of ARIP2. Interestingly, unlike ARIP2, overexpression of ARIP2b or $2 c$ did not affect ActRIIA internalization. ARIP $2 b / 2 c$ inhibit inhibitory actions of ARIP2 on activin signaling. ARIP2 is widely distributed in mouse tissues. ARIP2b/2c is expressed in more restricted tissues such as heart, brain, kidneys and liver. Our results indicate that although both ARIP2 and ARIP2b/2c interact with activin receptors, they regulate ActRIIA function in a different manner.

Journal of Endocrinology (2006) 189, 409-421

\section{Introduction}

Activins belong to the transforming growth factor- $\beta$ (TGF- $\beta$ ) superfamily, and signaling occurs via two types of membrane-bound receptor complexes (Attisano et al. 1996, Tsuchida et al. 2001). The two types of activin receptors, i.e. type I and type II, are serine/threonine kinase receptors. The two subtypes of the activin type II receptor, ActRIIA and IIB, are encoded by separate genes (Sugino \& Tsuchida 2000). In addition, two spliced variants of ActRIIA including ActRIIA-N (Mathews \& Vale 1991, Shoji et al. 1998), and five spliced variants of ActRIIB have been detected (Attisano et al. 1992, Ethier et al. 1997). Ligands that bind to ActRIIs include activins, myostatin, nodal and bone morphogenetic protein 7 (BMP-7). Direct binding of these ligands to ActRIIs promotes the recruitment of type I receptors to the complex, which in turn results in phosphorylation of type I receptors, activation of type I receptors, and downstream propagation of the signal (Mathews 1994, Sugino \& Tsuchida 2000, Tsuchida 2004).

The significance of the different ActRIIs is yet to be determined. Signal transduction via transmembrane receptors is regulated by their interaction with PDZ domain-containing molecules. PDZ domain-containing proteins play an important role in assembly of receptors and signaling molecules near submembranous regions (Fanning \& Anderson 1999). To determine the specific functions of ActRIIs in regulating activin signal transduction, we searched for cytoplasmic proteins that interacted with the receptors. We identified two cytoplasmic proteins named activin receptor-interacting proteins 1 and 2 (ARIP1 and ARIP2). ARIP1 showed multiple proteinprotein interacting domains including two WW domains which interacted with Smad3, and five to six PDZ domains which interacted with ActRIIs, phosphatase and 
tensin homologue deleted on chromosome 10 (PTEN) and $\beta$-catenin (Hirao et al. 1998, Shoji et al. 2000). ARIP2 possesses one PDZ domain which interacts with ActRIIA. ARIP2 also associates with Ral binding protein 1 (RalBP1), and assembles into a ternary complex that includes ARIP2, ActRII and RalBP1 (Matsuzaki et al. 2002). Overexpression of ARIP1 and ARIP2 inhibits activin-induced transcriptional activity in a dosedependent manner (Shoji et al. 2000, Matsuzaki et al. 2002). In the present study, we report the identification of two isoforms of ARIP2, named ARIP2b and 2c. Similar to ARIP2, ARIP2b and $2 c$ have only one PDZ domain, which specifically interacts with ActRIIA. However, even though ARIP2b and $2 \mathrm{c}$ were structural variants of ARIP2, they showed different functions. Overexpression of ARIP2b and $2 c$ resulted in increased activin-induced signaling. Furthermore, we found that ARIP2b and 2c increased ActRIIA expression at the cell surface without affecting internalization of ActRIIA, whereas ARIP2 was involved in endocytosis of ActRIIA (Matsuzaki et al. 2002). Thus, multiple ARIP2 proteins can interact with ActRII to regulate its signaling and trafficking in a different manner.

\section{Materials and Methods}

\section{cDNA cloning}

Yeast two-hybrid screening was performed using a commercially available system (Matchmaker Two-Hybrid System 2; Clontech) in accordance with the manufacturer's protocol. Approximately $4 \times 10^{6}$ clones of a mouse brain cDNA library were screened using the bait construct pAS-ActRIIA, which contains nucleotide sequences for the entire cytoplasmic region of mouse ActRIIA (5541995) fused to a GAL4 DNA binding domain (Shoji et al. 2000, Tsuchida et al. 2001). One clone obtained by the yeast two-hybrid screening was named \#YA-1. A mouse brain cDNA library in the lambda ZAPII vector (Stratagene, La Jolla, CA, USA) was screened using the \#YA-1 cDNA as probe. ARIP2 cDNA was identified by screening as described previously (Matsuzaki et al. 2002). Additional clones encoding full-length ARIP2b and $2 c$ sequences were identified by screening the brain library using full-length ARIP2 cDNA as hybridization probe.

\section{DNA constructs}

DNA constructs for the yeast two-hybrid screening were made using either the plasmid pAS2-1 to express the fusion protein with the GAL4 DNA-binding domain, or the plasmid pACT2 to express the fusion protein with the GAL4 activation domain. pAS-ActRIIA was made by introducing nucleotides 554-1995 of mouse ActRIIA (Mathews \& Vale 1991) into pAS2-1. For the mammalian two-hybrid assay, DNA was constructed using the plasmid pBIND to express the fusion protein with the GAL4 DNA-binding domain, and the plasmid pACT to express the fusion protein with the VP16 activation domain. To make pBIND-ActRIIA, cDNA fragments encoding the entire cytoplasmic regions of ActRIIA were subcloned into pBIND. Using PCR-based mutagenesis, mutations in the $\mathrm{COOH}$ terminus of ActRIIA were generated by replacing appropriate nucleotides with mutated oligonucleotides (Shoji et al. 2000). Other receptor constructs have been described previously (Shoji et al. 2000). To make pACT-ARIP2b and 2c, cDNA fragments covering coding regions (nucleotides 453-758 of ARIP2b, and nucleotides 1-357 of ARIP2c) were prepared and subcloned into pACT. To make pACT-ARIP2c $\Delta C$ and pACT-ARIP2c $\triangle P D Z, c D N A$ fragments composed of nucleotides 1-297 and 298-357 of ARIP2c respectively, were prepared by PCR and ligated into pACT. Expression constructs of ARIP2b and $2 c$ were made by subcloning nucleotides 453-758 of ARIP2b and nucleotides 1-357 of ARIP2c into pCIneo and pcDNA3 respectively. FLAG-tagged ARIP2b and $2 c$ were subcloned into pcDNA3. Full length ActRIIA cDNA was subcloned into pcDLSR $\alpha$. Myc-tagged ActRIIA cDNA in pcDNA3 was made by incorporating 7 myc epitopes after the arginine residue (amino acid number 24) of ActRIIA cDNA in pcDNA3.

\section{Yeast and mammalian two-hybrid analysis}

Yeast two-hybrid assays were performed using a commercially available kit (Matchmaker Two-Hybrid System 2; Clontech) in accordance with the manufacturer's protocol. Mammalian two-hybrid assays were performed using the CheckMate Mammalian Two-Hybrid System (Promega). In brief, Chinese hamster ovary K1 (CHO-K1) cells were co-transfected with appropriate plasmids, a cytomegalovirus promoter-driven $\beta$ galactosidase (CMV- $\beta$-gal), and the reporter plasmid pG5 luc, which drives the luciferase gene under the control of the GAL4-responsive promoter. Luciferase activity was measured and normalized against the level of $\beta$-gal activity as described previously (Tsuchida et al. 1995).

\section{Immunoprecipitation and Western blotting}

Interactions of full-length ARIP2b or $2 \mathrm{c}$ with ActRIIA were studied. Using the calcium phosphate precipitation method, COS-7 cells or HEK 293 cells were transfected either with pcDNA3-FLAG-ARIP2b or $2 c$ alone or co-transfected with pcDNA3-FLAG-ARIP2b (or 2c) and pcDLSR $\alpha$-ActRIIA. Two days after transfection, cells were harvested using lysis buffer (1\% Nonidet P-40, $50 \mathrm{mM}$ Tris- $\mathrm{HCl}$ (pH 7.5), $150 \mathrm{mM} \mathrm{NaCl}, 1 \mathrm{mM} \mathrm{NaF}$, $1 \mathrm{mM}$ phenylmethylsulfonyl fluoride (PMSF), $4 \mu \mathrm{g} / \mathrm{ml}$ leupeptin, and $1 \mu \mathrm{g} / \mathrm{ml}$ aprotinin). The lysate was cleared 
by centrifugation, and proteins were immunoprecipitated by adding a rabbit anti-ActRIIA polyclonal antibody (R\&D Systems, Minneapolis, MN, USA), or anti-FLAG M2 monoclonal antibody (Sigma). Samples were then incubated with protein G-sepharose (Amersham Pharmacia Biotech) at $4{ }^{\circ} \mathrm{C}$ for $2 \mathrm{~h}$. Precipitated proteins were fractionated by SDS-PAGE, and transferred onto a polyvinylidene difluoride (PVDF) membrane. Membranes were initially incubated with an anti-FLAG antibody (1:1000 dilution), and then with a horseradish peroxidaseconjugated secondary antibody. Labeled proteins were detected by chemiluminescence (ECL-Plus; Amersham Pharmacia Biotech) (Bao et al. 2005). To study interactions of ARIP2 or ARIP2c with RalBP1, FLAG-ARIP2 (or 2c) and myc-tagged RalBP1 were co-transfected into HEK 293 cells and the lysate was immunoprecipitated with an anti-FLAG antibody and immunodetected using myc antibody (1:1000 dilution).

\section{RNA extraction and RT-PCR}

Total RNA from mouse tissues was extracted using the TRIzol reagent according to the manufacturer's protocol (Invitrogen). Polyadenylated RNA was further purified using oligo (dT) latex beads (Takara, Tokyo, Japan). RNA samples were then reverse transcribed with oligo dT primer using SUPERSCRIPT II reverse transcriptase (Gibco-BRL). The ARIP2 primer set included the sense primer 5'-GGA GAG CAG TCA GAT ATG AAC G-3', and the antisense primer $5^{\prime}$-CAC GAA GAC CAA AAG AAC CTC CAA C-3', and the ARIP2c primer set included the sense primer $5^{\prime}$-GGA GAG CAG TCA GAT ATG AAC G-3', and the antisense primer 5'-CTA CTG TCC CAT ATC CAG GTG C-3'. PCR was performed for 28 cycles, with each cycle consisting of $94^{\circ} \mathrm{C}$ for $30 \mathrm{~s}, 60^{\circ} \mathrm{C}$ for $30 \mathrm{~s}$ and $72{ }^{\circ} \mathrm{C}$ for $30 \mathrm{~s}$, followed by a final extension step at $72{ }^{\circ} \mathrm{C}$ for $8 \mathrm{~min}$. Amplified PCR products were subjected to $2 \%$ agarose gel electrophoresis, and were stained using ethidium bromide for detection.

\section{Antibody preparation and Western blotting}

Glutathione S-transferase (GST) fusion proteins of fulllength ARIP2b (amino acids 1-101) were used for immunization of New Zealand White rabbits. To obtain polyclonal antibodies recognizing the $\mathrm{COOH}$-terminal region of ARIP2b/2c, but not ARIP2 and other PDZ proteins, crude rabbit antibodies were purified using protein A-Sepharose 4B (Amersham Biosciences), and affinity-chromatography of GST fusion proteins with N-terminal amino acids 1-82 of ARIP2b. The antibodies that recognize the COOH-terminal region of ARIP2b/ 2c, but not ARIP2, were thus obtained and used for Western blot analysis. Multiple mouse tissues were homogenized in buffer (1\% Triton X-100, $50 \mathrm{mM}$ Tris$\mathrm{HCl}(\mathrm{pH} 7 \cdot 5), 150 \mathrm{mM} \mathrm{NaCl}, 1 \mathrm{mM}$ PMSF, $4 \mu \mathrm{g} / \mathrm{ml}$ leupeptin, $1 \mu \mathrm{g} / \mathrm{ml}$ aprotinin), and centrifuged to yield supernatant fractions. Proteins were immunoprecipitated by adding an anti-ARIP $2 \mathrm{~b} / 2 \mathrm{c}$ polyclonal antibody to the supernatant, and then incubated with protein $\mathrm{G}$-sepharose (Amersham Pharmacia Biotech) at $4{ }^{\circ} \mathrm{C}$ for $2 \mathrm{~h}$. Precipitated proteins were fractionated by SDS-PAGE using a $15 \%$ gel, and transferred onto a PVDF membrane. Membranes were initially incubated with an anti-ARIP2b/2c polyclonal antibody (diluted 1:500), and then with a horseradish peroxidase-conjugated secondary antibody. As a loading control, membranes were also probed with anti-actin antibody (Sigma).

\section{Activin-responsive promoter analysis}

The CAGA-lux construct has been described previously (Dennler et al. 1998). CAGA-lux, CMV- $\beta$-gal, pCIneo-ARIP2b and pcDNA3-ARIP2c were introduced into HEK 293 cells or L $\beta$ T2 cells using TransFast liposome reagents (Promega) in accordance with the manufacturer's protocol. Stimulation by activin A and measurement of luciferase activity were performed as described previously (Tsuchida et al. 1995). We used activin A from WAKO Chemicals (Osaka, Japan) that is fully active.

\section{Radioimmunoassay for follicle stimulating hormone (FSH)}

L $\beta$ T2 cells were grown in 12-well plates in DMEM supplemented with $10 \%$ fetal calf serum (FCS). ARIP2b cDNA was introduced into L $\beta$ T2 cells using TransFast liposome reagents. Stimulation by $50 \mathrm{ng} / \mathrm{ml}$ activin A and measurement of FSH levels in conditioned media by radioimmunoassay (RIA) were performed as previously described (Graham et al. 1999). The sensitivity of the RIA was $4 \mathrm{ng} / \mathrm{ml}$.

\section{Cell surface ${ }^{125}$ I-activin binding and internalization assay}

CHO-K1 cells grown in 6-well plates were transfected with ActRIIA cDNA with or without pcDNA3-ARIP2c using TransFast liposome reagents. To examine total (cell surface and internalized) activin binding activity of cells, transfected cells were washed three times with cold binding medium (minimum essential medium containing $20 \mathrm{mM}$ HEPES-NaOH $(\mathrm{pH} 7 \cdot 4)$ and $0 \cdot 1 \%$ bovine serum albumin), and incubated in $800 \mu$ cold binding medium containing $15 \mathrm{ng}{ }^{125}$ I-activin A labeled by the chloramine T method (Matsuzaki et al. 2002) for the indicated time periods on ice. Then unbound ligands were removed by washing three times with cold PBS. Cells were solubilized in $1 \mathrm{~N} \mathrm{NaOH}$, and the radioactivities of the cells were counted in a $\gamma$ counter. To study internalization of ActRIIA, transfected cells were washed three times with binding medium, and incubated in $800 \mu \mathrm{l}$ binding medium containing $15 \mathrm{ng}{ }^{125} \mathrm{I}$-activin $\mathrm{A}$ at $37^{\circ} \mathrm{C}$ for the 
appropriate time periods. Following incubation, plates were placed on ice, washed three times with cold PBS, and then incubated with cold acid washing buffer $(0 \cdot 2 \mathrm{M}$ acetic acid and $0.5 \mathrm{M} \mathrm{NaCl}$ ) for $5 \mathrm{~min}$ on ice. Then, cells were solubilized in $1 \mathrm{M} \mathrm{NaOH}$, and the radioactivities in the lysates were counted as above. Cell-associated radioactivity after the acid wash represented internalized activin mainly through receptor binding. Internalization was determined as the ratio of internalized ${ }^{125}$ I-activin-A/ surface bound ${ }^{125}$ I-activin-A. Nonspecific binding and internalization activities were determined in the presence of a 100-fold excess of unlabeled activin A.

\section{Determination of ActRIIA expression at the cell surface}

HEK 293 cells in 6-well dishes were transfected with myc-tagged ActRIIA cDNA and/or pcDNA3-ARIP2c expression plasmids, then cells were harvested in $200 \mu \mathrm{l}$ PBS at various time points after transfection. Cells were then treated with $3 \mu \mathrm{l}$ rabbit anti-myc antibody at $4{ }^{\circ} \mathrm{C}$ for $2 \mathrm{~h}$ to label ActRIIA at the cell surface, washed three times with PBS, and then extracted with lysis buffer as described in the Materials amd Methods section. Labeled receptors were collected with protein A sepharose, and detected by blotting with anti-ActRIIA antibody as described above.

Quantitative measurement of cell surface ActRIIA associated with ARIP2

HEK 293 cells were co-transfected with myc-tagged ActRIIA, FLAG-ARIP2 and/or FLAG-ARIP2c cDNAs. To quantitate ARIP2s that associate with ActRIIA, cell proteins were extracted with lysis buffer and immunoprecipitated with anti-myc antibody, and co-precipitated ARIP2s were detected by blotting with anti-FLAG antibody. To detect cell surface ActRIIA associated with ARIP2s, myc-tagged ActRIIA at the cell surface was labeled with myc antibody, collected by protein A sepharose and blotted with anti-FLAG antibody as described above.

\section{Detection of recycling ActRIIA associated with ARIP2}

HEK 293 cells were transfected with myc-tagged ActRIIA, FLAG-ARIP2 and FLAG-ARIP2c cDNAs. Twenty-four hours after transfection, cells were washed with PBS and exposed to $0.025 \%$ trypsin for $10 \mathrm{~min}$ at $4{ }^{\circ} \mathrm{C}$. At the end of trypsin treatment, cells were washed three times with medium containing 10\% FCS to stop trypsin activity. Then, cells were re-incubated in medium with $10 \% \mathrm{FCS}$ at $37{ }^{\circ} \mathrm{C}$, and treated either with $500 \mathrm{mM}$ chloroquine or $50 \mathrm{mM}$ monensin from WAKO Chemicals. Cells were collected at appropriate times. Cell surface myc-ActRIIA was labeled with myc antibody, collected with protein A sepharose and blotted with
anti-FLAG antibody to detect ARIP2s as described above. To detect recycling ActRIIA associated with ARIP2s, total lysate was immunoprecipitated with myc antibody, then co-precipitated ARIP2s were detected by blotting with anti-FLAG antibody.

\section{Results}

\section{Identification of isoforms of ARIP2}

In the search for intracellular proteins that interact with ActRIIs, we obtained about 30 positive clones, including ARIP1 and ARIP2, with the yeast two-hybrid screening. Association of ARIP1 and ARIP2 with ActRIIA in mammalian cells was verified by co-immunoprecipitation and mammalian two-hybrid analysis (Shoji et al. 2000, Matsuzaki et al. 2002). Screening of a mouse brain cDNA library with the $462 \mathrm{bp}$ coding region of ARIP2 as hybridization probe yielded full length cDNA clones of ARIP2b and 2c, and ARIP2 (Fig. 1A). ARIP2 encoded a protein of 153 amino acids including the single PDZ domain as reported previously (Matsuzaki et al. 2002). ARIP2b cDNA encoded a protein of 101 amino acids with one PDZ domain which was identical to that of ARIP2 except for 8 amino acids at the $\mathrm{NH}_{2}$-terminal. However, the ARIP2b amino acid sequences differed from ARIP2 outside the PDZ domain (Fig. 1A,B). ARIP2c is composed of 118 amino acids, and is completely identical to ARIP2 from the $\mathrm{NH}_{2}$-terminal region through the PDZ domain, but its $\mathrm{COOH}$-terminal region is identical with that of ARIP2b (Fig. 1A).

\section{ARIP2b and $2 c$ interact specifically with ActRIIA among serine/threonine kinase receptors}

PDZ domains recognize a class I consensus PDZ-binding motif $\mathrm{XSX}(\mathrm{V} / \mathrm{I} / \mathrm{L})$ (where $\mathrm{X}$ is any amino acid) in the $\mathrm{COOH}$ terminus of target proteins (Songyang et al. 1997). In agreement with the consensus PDZ-binding sequence, the four COOH-terminal amino acids of ActRIIA were ESSL. ARIP2b interacted with ActRIIA, but did not associate with ActRIIB, TGF- $\beta$ receptor type II (TGFßRII) or BMP receptor type II (BMPRII) by mammalian two hybrid analysis (Fig. 2A). Like ARIP2b, ARIP2c also interacted with ActRIIA. ARIP2c did not interact with mutated ActRIIA proteins that lacked either the $\mathrm{COOH}$-terminal SSL or $\mathrm{COOH}$-terminal leucine (Fig. 2B). Similar results were obtained for ARIP2b (data not shown). Thus, $\mathrm{COOH}$-terminal amino acids of ActRIIA play a key role in interactions between ARIP2b and ARIP2c via the PDZ domain. Amino acid sequences of target proteins relate to specific interactions with various PDZ proteins. It is worthwhile noting that although ARIP2 interacted with both ActRIIA and ActRIIB, ARIP2b and 2c interacted with ActRIIA, 


\begin{abstract}
(A)
\begin{tabular}{ll|l|l|} 
ARIP2 1 MNGRVDYLVTEEEINLTRGPSGLGFNIVGGTDQQYVSNDSGIYVSRIKEDGAAAQDGRLQ & 60
\end{tabular}

\begin{tabular}{ll|l|l|l|} 
ARIP $2 \mathrm{~b}$ & 1 & M---I-F----------SGLGFNIVGGTDQQYVSNDSGIYVSRIKEDGAAAQDGRLQ & 43
\end{tabular}

\begin{tabular}{ll|l|l} 
ARIP2c & 1 MNGRVDYLVTEEEINLTRGPSGLGFNIVGGTDQQYVSNDSGIYVSRIKEDGAAAQDGRLQ & 60
\end{tabular}

ARIP2 61 EGDKILSVNGQDLKNLLHQDAVDLFRNAGCAVSLRVQHRLLVVGGSFGLREFSQIRYDAV 120

ARIP2b 44 EGDKILSVNGQDLKNLLHQDAVDLFRNAGCAVSLRVQHR-VGITCTW-IWD-SRLLHCSC 100

ARIP2C 61 EGDKILSVNGQDLKNLLHQDAVDLFRNAGCAVSLRVQHR-VGITCTW-IWD-SRLLHCSC 117

ARIP2 121 TIKIDPELEKKLKVNKITLESEYERLLCLLCRQ 153

ARIP2b 101 E---D-- 101

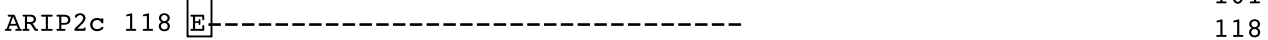

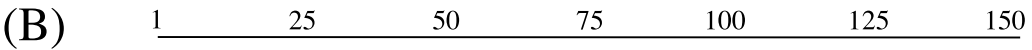

ARIP2 PDZ domain

ARIP2b

PDZ domain

ARIP2c

PDZ domain

Figure 1 Identification of ARIP2 isoforms. (A) Alignment of amino acid sequences of ARIP2 and its two isoforms, ARIP2b and ARIP2c. PDZ domains are indicated in bold letters. Identical amino acids are boxed. (B) Schematic illustration of protein structures of ARIP2 along with those of ARIP2b and 2c. The $\mathrm{NH}_{2}$-terminal amino acid residues in ARIP2b that differ from ARIP2 are shown as a white box. The $\mathrm{COOH}$-terminal amino acid residues unique in ARIP2 are indicated as a white box. The $\mathrm{COOH}$-terminal amino acid residues in ARIP2b/2c that differ from ARIP2 are shown as gray boxes.
\end{abstract}

but not with ActRIIB (Fig. 2A,B). These results indicate that ARIP2b or $2 \mathrm{c}$ interact specifically with ActRIIA among type II serine/threonine kinase receptors of the TGF- $\beta$ superfamily, and that this interaction occurs in both yeast and mammalian cells. We then studied which part of ARIP2c interacted with ActRIIA using a mammalian two-hybrid method. ARIP2c $\Delta \mathrm{C}$, but not ARIP2c $\Delta \mathrm{PDZ}$ interacted with ActRIIA, indicating that ARIP2c interacts with ActRIIA through a PDZ domain-mediated interaction (Fig. 2C). ARIP2b, 2c and ARIP2 have an identical amino acid sequence in the PDZ domain which is important for interactions with ActRIIA. Lysates from COS-7 cells that have been co-transfected with ActRIIA, and FLAG-tagged ARIP2c were incubated with an antiActRIIA antibody, and co-immunoprecipitated ARIP2c protein was detected by an anti-FLAG antibody. As shown in Fig. 2D, ActRIIA and ARIP2c formed a protein complex in transfected cells. Similar interactions of ActRIIA and ARIP2b were observed (data not shown). ARIP1 interacts with Smads via WW domains, whereas ARIP2 does not interact with Smad proteins (Shoji et al. 2000, Matsuzaki et al. 2002). ARIP2 isoforms, i.e. ARIP2b and 2c, like ARIP2, did not show any interaction with Smad (data not shown).
Expression of ARIP2 isoforms in mouse tissues

Northern blot analysis of poly $(\mathrm{A})^{+} \mathrm{RNA}$ extracted from several mouse tissues using full length ARIP2 cDNA probe yielded two major bands of $4.5 \mathrm{~kb}$ and $1.1 \mathrm{~kb}$ (Matsuzaki et al. 2002). Since very short sequences in the $\mathrm{COOH}$-terminal of $\mathrm{ARIP} 2 \mathrm{~b} / 2 \mathrm{c}$ are different from ARIP2, and ARIP2 has an additional isoform called synaptojanin binding protein (also known as outer membrane protein of $25 \mathrm{kDa}$, OMP25) (Nemoto \& DeCamilli 1999), it is difficult to detect ARIP2b/2c by Northern blotting. Therefore, we compared the tissue distribution of ARIP2 and ARIP2c mRNAs using RT-PCR. As shown in Fig. 3A, both ARIP2 and ARIP2c are widely expressed in various mouse tissues. ARIP2 mRNA was detected in multiple tissues. ARIP2c mRNA was detected in brain, liver, kidney, ovary and testis Although ARIP2 and $2 \mathrm{c}$ are structurally similar, their distributions differ slightly. ARIP2b mRNA was detected in heart, spleen and testes by RT-PCR, but its expression levels were much lower than those of ARIP2c mRNA (data not shown). We also studied protein expression of ARIP2b/ 2c. Antibodies that recognized ARIP $2 b / 2 c$ but not ARIP2 or OMP25 were prepared and used for Western 
(A)

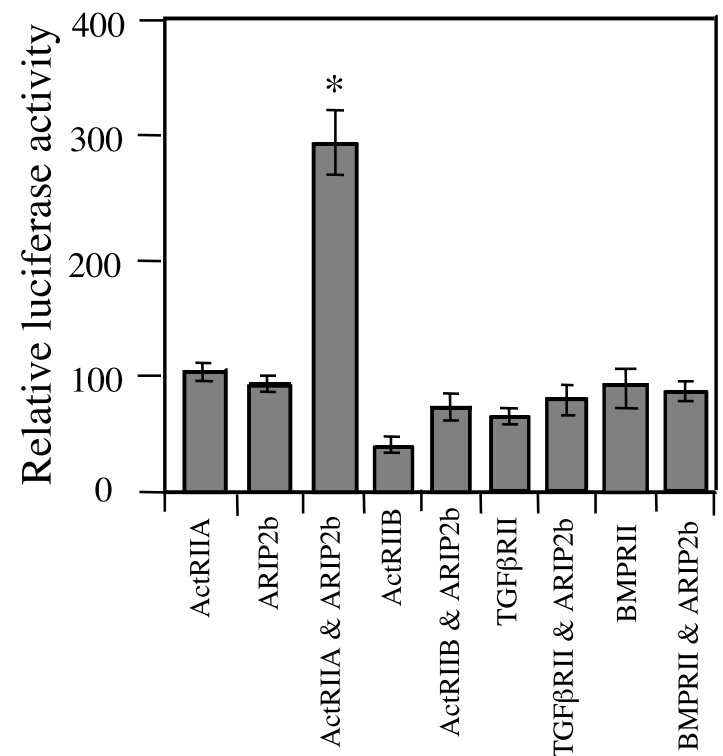

(B)

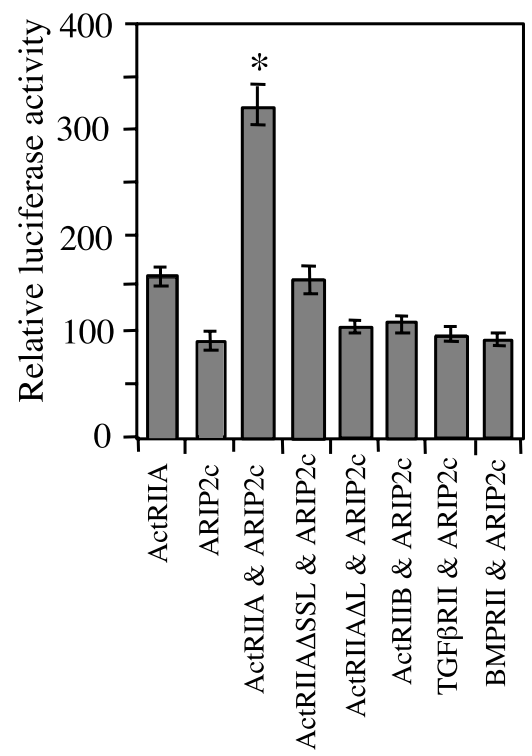

(C)

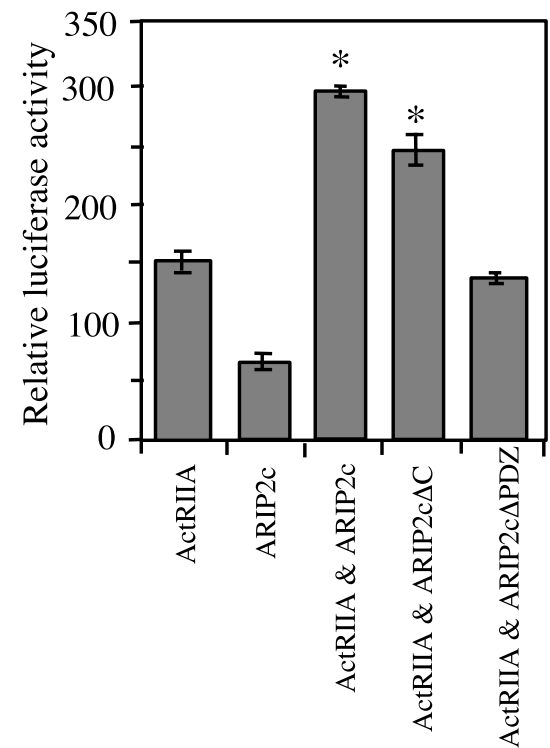

(D)

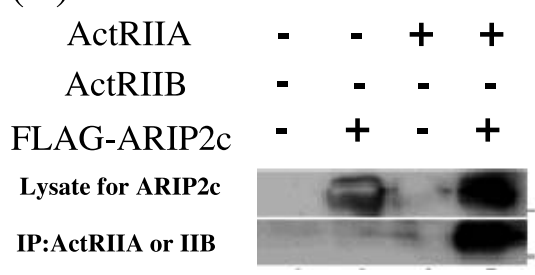

Lysate for IIB

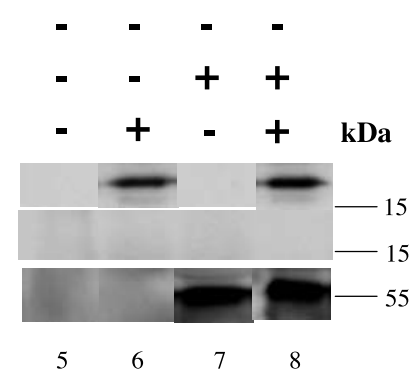

Figure 2 Interactions of ARIP2b or 2c with ActRIIA assessed by mammalian two-hybrid analysis. (A) Interaction of ARIP2b with ActRIIA, ActRIIB, TGF $\beta$ RII and BMPRII in CHO-K1 cells. Relative interaction is shown as luciferase activity. Expression vectors used were pACT-ARIP2b and pBIND plasmids, into which the entire cytoplasmic region of each receptor was introduced. Values in the figure represent means and S.D. of triplicate determinations. ${ }^{*} P<0 \cdot 01$ vs ActRIIA using a $t$-test. (B) Interaction of ARIP2C with ActRIIA, ActRIIA $\triangle S S L, A c t R I I A \Delta L, A c t R I I B, T G F \beta R I I$ and BMPRII in CHO-K1 cells. Relative interaction is represented as luciferase activity. ${ }^{*} P<0.01$ vs ActRIIA using a $t$-test. (C) Interaction of ARIP2c, ARIP2c $\Delta C$ or ARIP2c $\Delta P D Z$ with ActRIIA in CHO-K1 cells. Relative interaction is represented as luciferase activity. Values in the figure represent means and S.D. of triplicate determinations. ${ }^{*} P<0 \cdot 01 \mathrm{vs}$ ActRIIA using a t-test. (D) Interaction of ActRIIA with ARIP2c by immunoprecipitation (IP). In lanes 1-4, lysates of COS-7 cells transfected with either pcDNA3-FLAG-ARIP2c (lane 2), or pcDLSR $\alpha$-ActRIIA (lane 3), or both (lane 4), or untransfected (lane 1) were immunoprecipitated with an anti-ActRIIA antibody, and probed with an anti-FLAG monoclonal antibody (bottom row). On the top row, transfected COS-7 cells were probed with an anti-FLAG antibody. The molecular weight marker is indicated on the right. In lanes 5-8, COS-7 cells transfected either with pcDNA3-FLAG-ARIP2c (lane 6) or pcDLSR $\alpha$-ActRIIB (lane 7) or both (lane 8), or untransfected (lane 5) were immunoprecipitated with an anti-ActRIIB antibody, and probed with an anti-FLAG monoclonal antibody (middle row). To show expression of ActRIIB, lysates were probed with ActRIIB antibody in the bottom row. 
(A)

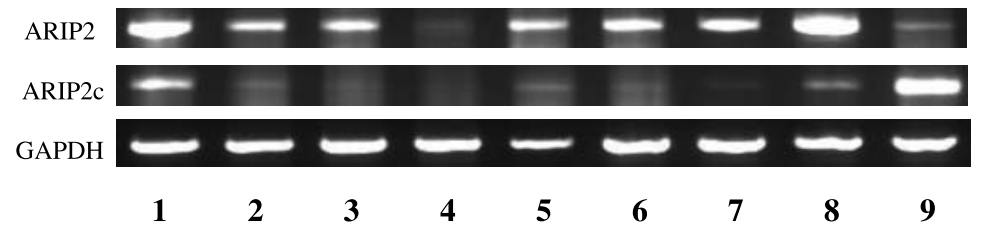

(B)

$\mathrm{ARIP} 2 \mathrm{~b} / 2 \mathrm{c}$

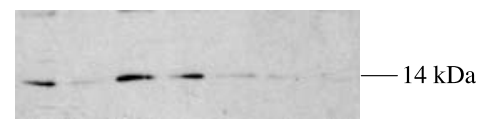

Actin

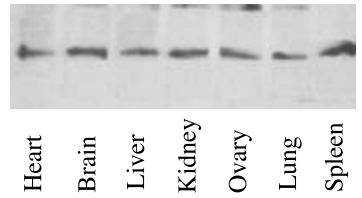

Figure 3 Distribution of ARIP2 isoforms in mouse tissues. (A) Tissue distribution of ARIP2 and ARIP2c mRNAs. mRNA isolated from various mouse tissues were identified by RT-PCR analysis. For control, glyceraldehyde-3-phosphate dehydrogenase (GAPDH) mRNA was also amplified. Lane 1, brain; lane 2, heart; lane 3, lung; lane 4, spleen; lane 5, liver; lane 6, kidney; lane 7, skeletal muscle; lane 8, ovary; lane 9, testis. (B) Tissue distribution of ARIP2b/2c by Western blot analysis. A polyclonal antibody recognizing the $\mathrm{COOH}$-terminus of ARIP2b/2c was used for detection (top row). For control, antibody to actin was used (bottom row). The molecular weight marker is indicated on the right.

blot analysis. As shown in Fig. 3B, ARIP2b/2c signal was present in heart, liver and kidneys, and to a lesser extent in brain, ovaries and lungs.

\section{ARIP2b/2c has a stimulatory effect on activin-induced signaling and secretion of FSH from L $\beta$ T2 cells}

HEK 293 cells were co-transfected with ARIP2c cDNA and the reporter plasmid CAGA-lux, and activin-induced luciferase activity was measured. CAGA-lux is a reporter plasmid that has CAGA tandem repeats, and responds well to activin and TGF- $\beta$ stimuli (Dennler et al. 1998). Overexpression of ARIP2c augmented activin-induced transcriptional activity in a dose-dependent manner (Fig. 4A). We also studied the effects of ARIP2b on FSH secretion in gonadotroph L $\beta$ T2 cells, which secrete FSH in response to activin (Graham et al. 1999). L $\beta$ T2 cells were transfected with ARIP2b and CAGA-lux DNAs, and activin-induced luciferase activity was measured. Overexpression of ARIP2b cDNA increased activity of CAGA promoter in a dose-dependent manner (Fig. 4B). FSH secretion by activin in the culture media was actually induced by ARIP2b cDNA transfection in L $\beta$ T2 cells (Fig. 4C). ARIP2c showed a similar activity to ARIP2b.

\section{Antagonism of ARIP2 and ARIP2b on activin signaling}

In a previous report, we showed that ARIP2 inhibited activin signaling (Matsuzaki et al. 2002). Since ARIP2 and ARIP2b/2c have opposite effects on activin signaling, we investigated whether ARIP2 and ARIP2b have antagonistic activities when expressed simultaneously. Expression of ARIP2b cDNA augmented activin signaling in a dosedependent manner in HEK 293 cells (Fig. 4D). In the presence of ARIP2, activin signaling was inhibited. When ARIP2b was co-expressed with ARIP2, the inhibitory effect of ARIP2 was blocked by ARIP2b cDNA in a dose-dependent manner. This result indicates that ARIP2 and ARIP2b have antagonistic activities on activin signaling. ARIP2c, like ARIP2b, showed antagonistic activities to ARIP2 (data not shown). Since ARIP2b and $2 \mathrm{c}$ are expressed in numerous tissues, they could play a role in controlling activin signaling transduction.

\section{ARIP2b/2c neither affects endocytosis of ActRIIA nor interacts with RalBP1}

In a previous study, we reported that ARIP2 regulated endocytosis of ActRIIA through the Ral/RalBP1dependent pathway (Matsuzaki et al. 2002). To study the role of ARIP $2 b / 2 c$ in internalization of activin through ActRIIA, we performed an internalization experiment. Unlike ARIP2, overexpression of ARIP2c did not affect internalization of ActRIIA (Fig. 5A). In a parallel experiment, we reproduced enhanced internalization of ActRIIA by ARIP2 expression. To examine interactions of ARIP2c with RalBP1, we performed an immunoprecipitation experiment. As shown in Fig. 5B, ARIP2c did not significantly interact with RalBP1, whereas ARIP2 interacted with RalBP1. ARIP2b, like ARIP2c, did not intereact with RalBP1 (data not shown).

\section{ARIP2b/2c increases expression of ActRIIA on cell surfaces}

To further investigate interactions between ARIP2c and ActRIIA, we co-expressed these proteins and examined the effects on surface receptor expression levels. Figure 6A shows the effects of activity of ARIP2c on the expression of ActRIIA in HEK 293 cells. In the presence of ARIP2c, the number of ActRIIA at the cell surface increased faster after the $18 \mathrm{~h}$ time point compared with control (Fig. 6A). In contrast, in the presence of ARIP2, cell surface levels of ActRIIA did not significantly change when compared with control (Fig. 6B).

Mode of association of ARIP2c with ActRIIA is different from that of ARIP2 with ActRIIA

We next quantitated the amounts of ARIP2c and ARIP2 that associated with cell surface ActRIIA. After $6 \mathrm{~h}$ 

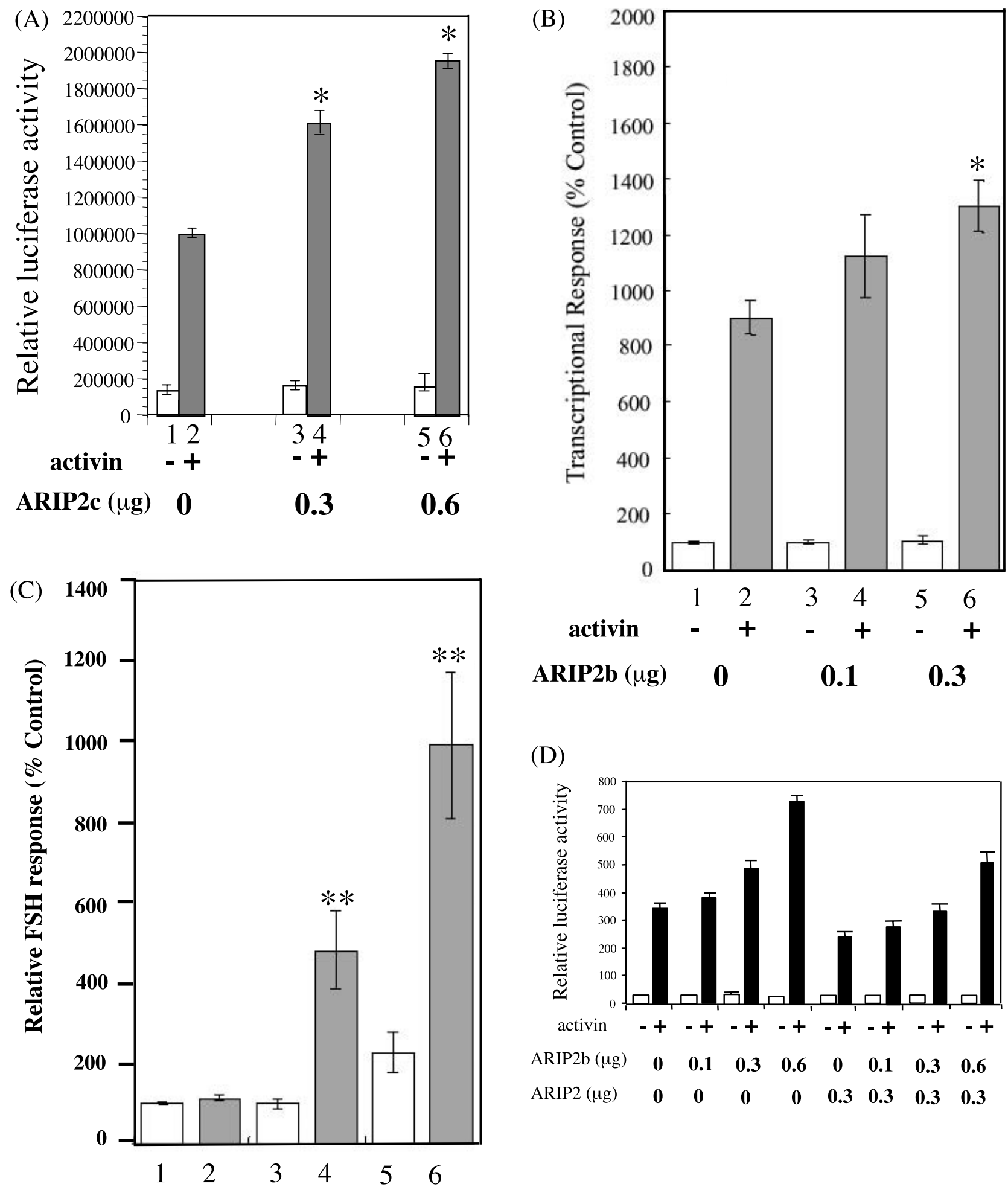

(D)

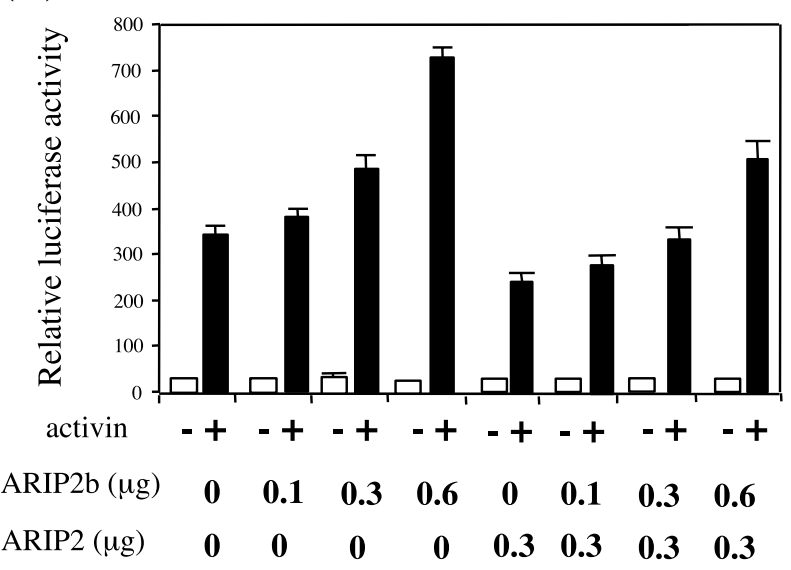

activin - $+\quad-+\quad-+$
ARIP2b $(\mu \mathrm{g}) \quad 0$
0.1
0.3 


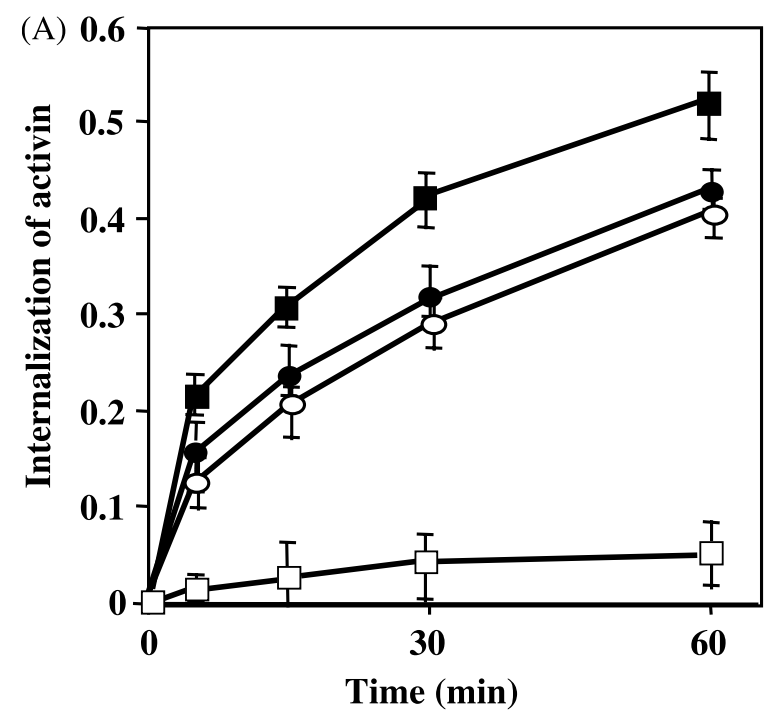

(B)

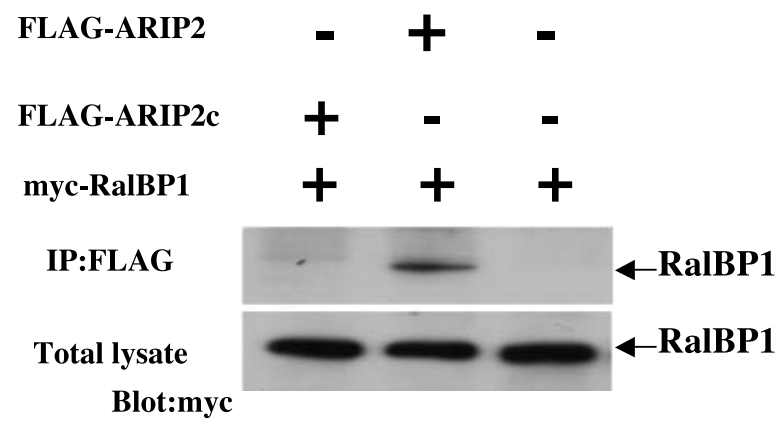

Figure 5 Effects of ARIP2C on ActRIIA internalization. (A) CHO-K1 cells were transfected with control vector $(\square)$, ActRIIA (O), ActRIIA and ARIP2C ( ) or ActRIIA and ARIP2 ( $)$, treated in binding buffer with $15 \mathrm{ng}{ }^{125}$ I-activin A, and incubated at $37{ }^{\circ} \mathrm{C}$ for appropriate time periods. Graphs show internalization of ActRIIA calculated using the ratio of internalized activin/surface activin binding as described in Materials and Methods. Values shown are means and S.D. of triplicate determinations. (B) Interaction of RalBP1 with ARIP2 or ARIP2c. COS-7 cells were transfected with myc-RalBP1 and FLAG-ARIP2 or FLAG-ARIP2c, and then lysates were immunoprecipitated (IP) with anti-FLAG antibody, separated by SDS-PAGE, blotted onto a membrane, and probed with anti-myc antibody. In the middle row, total lysate was analyzed for RalBP1 expression. In the bottom row, total lysate was analyzed for FLAG (F)-ARIP2 or F-ARIP2C expression.

transfection, ARIP2 and ARIP2c began to be expressed, then increased and reached a maximal value at $18 \mathrm{~h}$, and finally remained constant from 18 to $36 \mathrm{~h}$ after transfection (Fig. 6C, middle row). After $12 \mathrm{~h}$ transfection, ARIP2c co-precipitated with cell surface ActRIIA, and gradually reached a maximal amount from 18 to $36 \mathrm{~h}$. In contrast, ARIP2 co-precipitated with cell surface ActRIIA at a much slower rate than ARIP2c. After $18 \mathrm{~h}$ transfection, co-precipitation of ARIP2 was observed, and slowly increased thereafter (Fig. 6C, top row). We also investigated total (cell surface and intracellular) ActRIIA associating with ARIP2 or ARIP2c (Fig. 6D). ARIP2c co-precipitated with total ActRIIA after $6 \mathrm{~h}$ transfection, then increased to reach a maximal amount and then remained constant from 18 to $36 \mathrm{~h}$. In contrast,
ARIP2 co-precipitated with ActRIIA after $12 \mathrm{~h}$ transfection, then slowly increased. We studied differences in association mechanisms of ARIP2 and ARIP2c to cell surface ActRIIA. First, we trypsinized transfected HEK 293 cells to degrade cell surface receptor binding activities, and newly synthesized ActRIIA associating with ARIP2s was quantitated at various time points by coimmunoprecipitation. As shown in Fig. 7A, after $2 \mathrm{~h}$ trypsin stripping, cell surface receptor-associated ARIP2 and ARIP2c were observed, and increased rapidly. Almost the same amounts of ARIP2 and ARIP2c co-precipitated with cell surface ActRIIA (Fig. 7A). Next, we used cycloheximide to block new protein synthesis after trypsin treatment, and measured the amounts of ARIP2 and ARIP2c that bound to newly appearing ActRIIA at the

Figure 4 Effects of ARIP2b or 2c on activin-induced transcription. (A) ARIP2c-mediated increase in activin-induced transcription in HEK 293 cells. Cells were transfected with CAGA-lux, CMV- $\beta$-gal, and various amounts of ARIP2C cDNA, and then incubated with $50 \mathrm{ng} / \mathrm{ml}$ activin A for $12 \mathrm{~h}$. The level of luciferase activity of each cell lysate was measured, and normalized against the level of $\beta$-gal activity. Values in the figure represent means and S.D. of triplicate determinations. ${ }^{*} P<0 \cdot 01$ vs activin-treated cells without ARIP2c (lane 2) using a t-test. (B) Effects of ARIP2b on activin-induced transcription in L $\beta T 2$ cells. Cells were transfected with CAGA-lux, CMV- $\beta$-gal, and various amounts of ARIP2b cDNA, and then incubated with $50 \mathrm{ng} / \mathrm{ml}$ activin A for $12 \mathrm{~h}$. The level of luciferase activity of each cell lysate was measured, and normalized against the level of $\beta$-gal activity. Values in the figure represent means and S.D. of triplicate determinations. ${ }^{*} P<0.05$ vs activin-treated cells without ARIP2b (lane 2) using a $t$-test. (C) Activin-mediated increase in FSH secretion from L $\beta$ T2 cells. Cells were transfected with various amounts of ARIP2b cDNA, and incubated with $50 \mathrm{ng} / \mathrm{ml}$ activin A for $24 \mathrm{~h}$. FSH was assayed by a radioimmunoassay kit from NIDDK (Bethesda, MD, USA). FSH secretion from untransfected cells was $5 \cdot 0 \mathrm{ng} / \mathrm{ml}$, and was adjusted to 100 . ** $P<0 \cdot 01$ vs activin-treated cells without ARIP2b (lane 2) using a t-test. (D) Antagonist effects of ARIP2b and ARIP2 on activin signaling. HEK 293 cells were transfected with CAGA-lux, CMV- $\beta$-gal, and various amounts of ARIP2 and/or ARIP2b cDNA, and then incubated with $50 \mathrm{ng} / \mathrm{ml}$ activin A for $12 \mathrm{~h}$. The level of luciferase activity of each cell lysate was measured and normalized against the level of $\beta$-gal activity. Values in the figure represent means and S.D. of triplicate determinations. 
(A)

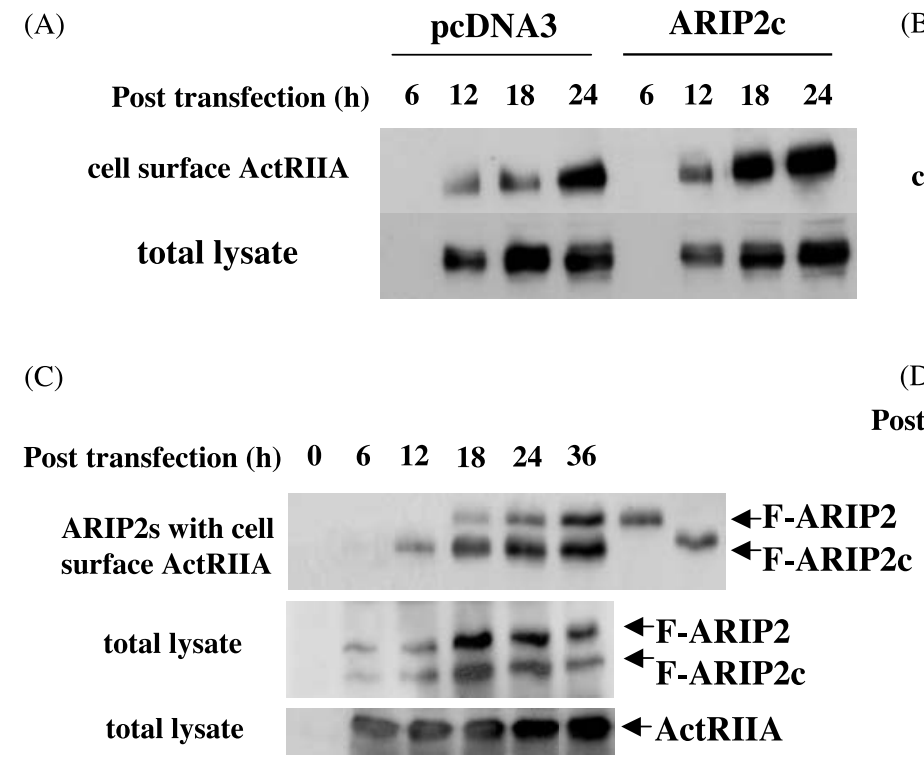

(D)
(B)

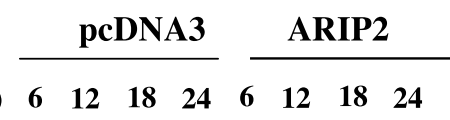

$\begin{array}{lllllllll}\text { Post transfection (h) } & 6 & 12 & 18 & 24 & 6 & 12 & 18 & 24\end{array}$

cell surface ActRIIA

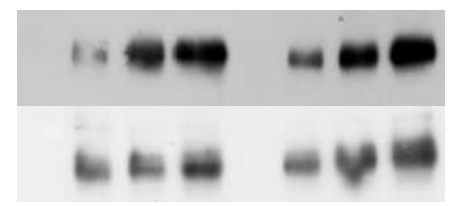

total lysate

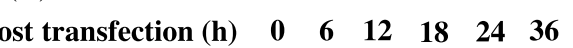

IP:myc WB:FLAG

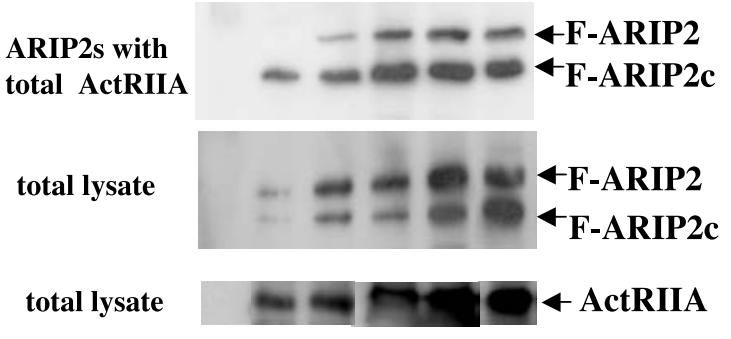

IP:myc WB:FLAG

Figure 6 Effects of ARIP2C on cell surface level of ActRIIA and association of ARIP2/ARIP2C with ActRIIA. (A) ARIP2C increases expression of ActRIIA at the cell surface. HEK 293 cells were transfected either with myc-tagged ActRIIA (left panel) or myc-tagged ActRIIA and ARIP2C CDNAs (right panel). After appropriate time periods, cells were harvested and treated with anti-myc antibody to label cell surface receptors, immunoprecipitated and detected by blotting with anti-ActRIIA antibody. (B) Same experiment as in (A) except that ARIP2 CDNA was used instead of ARIP2C CDNA. (C) HEK 293 cells were transfected with myc-tagged ActRIIA, FLAG (F)-ARIP2 and F-ARIP2c cDNAs. Then cell surface receptors were labeled with myc antibody, extracted, immunoprecipitated (IP), and blotted with FLAG antibody. In the middle and bottom rows, total lysate was analyzed. In the two rightmost lanes in the top row, positions of ARIP2 and ARIP2c are shown by comparison. (D) Same experiment as in (C) except that cell surface labeling with myc antibody was omitted to detect ARIP2s associating with total (cell surface and intracellular) ActRIIA. In the middle and bottom rows, total lysate was analyzed. WB, Western blotting.

plasma membrane (Fig. 7B). Although almost equal amounts of ARIP2 and ARIP2c were observed in the total lysate, only small amounts of ARIP2c that co-precipitated with cell surface ActRIIA were detected (Fig. 7B). This result indicates that ARIP2c, possibly acting in a dominant-negative manner to block ARIP2, is involved in blocking endocytosis of ActRIIA by ARIP2. Then, we studied the association of ARIP2 or ARIP2c with ActRIIA using the recycling inhibitors, chloroquine or monensin. When chloroquine was used to interfere with recycling after trypsin treatment, the amounts of cell surface ARIP2 associating with ActRIIA significantly decreased, whereas the presence of chloroquine did not significantly affect the amounts of ARIP2c co-precipitating with cell surface ActRIIA (Fig. 7C, left panel). When monensin was used to prevent recycling of the internalized receptor back to the cell surface, only a small amount of ARIP2 co-precipitated with cell surface ActRIIA (Fig. 7C, right panel). Even without trypsin treatment, chroloquine treatment affected the amounts of cell surface ActRIIA associated with ARIP2, whereas it did not significantly affect the amounts of ARIP2c co-precipitating with cell surface ActRIIA (Fig. 7D).
Taken together, these results indicate that ARIP2 is involved in endocytosis and recycling of ActRIIA, whereas ARIP2c, acting in a dominant-negative manner to block ARIP2, enhances ActRII levels at the cell surface.

\section{Discussion}

Activin type II receptors have been identified in multiple tissues, and are involved in signaling pathways of activins (Sugino \& Tsuchida 2000, Tsuchida 2004). Activins first bind to type II receptors, then type II receptors induce phosphorylation and activation of type I receptors. Then, type I receptors activate Smads to propagate signal transduction. ActRIIs retain a basal level of autophosphorylation that is independent of either type I receptors or ligand binding. Furthermore, type II receptors undergo endocytosis by adaptor proteins (Matsuzaki et al. 2002, Di Guglielmo et al. 2003). These findings suggest that type II receptors act as primary signal determinants. As a consequence, factors that control the actions of type II receptors regulate downstream propagation of activin-induced signaling. In the present study, we isolated and characterized 
(A)

\begin{tabular}{|c|c|c|c|c|c|}
\hline \multirow{2}{*}{$\begin{array}{l}\text { Post trypsin treatment (h) } \\
\text { ARIP2s with } \\
\text { cell surface } \\
\text { ActRIIA }\end{array}$} & $\mathbf{0}$ & 2 & 4 & 8 & \multirow[b]{2}{*}{$\begin{array}{l}{ }_{\text {-F-ARIP2 }} \\
{ }_{\text {F-ARIP2c }}\end{array}$} \\
\hline & & 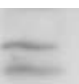 & 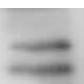 & 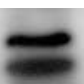 & \\
\hline total lysate & & 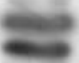 & 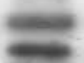 & 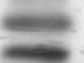 & $\begin{array}{l}\text { 4F-ARIP2 } \\
4 \text { - }\end{array}$ \\
\hline total lysate & & & & & $\leftarrow$ ActRIIA \\
\hline & & & WB:F & $\mathbf{G}$ & \\
\hline
\end{tabular}

(C)

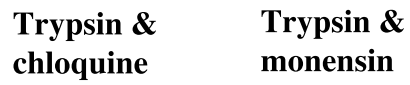

$\begin{array}{lllllllll}\text { Post trypsin treatment (h) } & 0 & 2 & 4 & 8 & 0 & 2 & 4 & 8\end{array}$
(B)

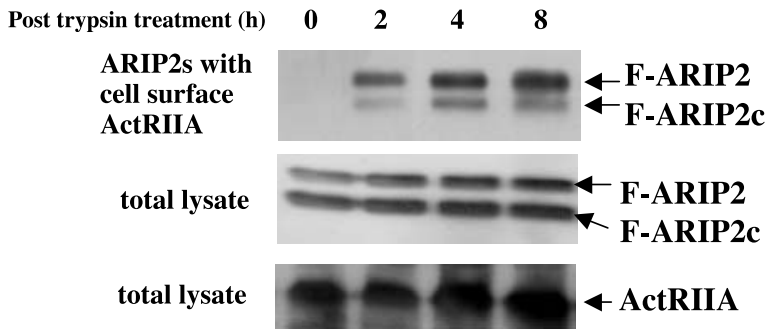

IP:myc WB:FLAG
(D) $\begin{array}{llll}\text { Post chloroquine treatment (h) } & 0 & 2 & 4\end{array}$

\section{IP:myc WB:FLAG}

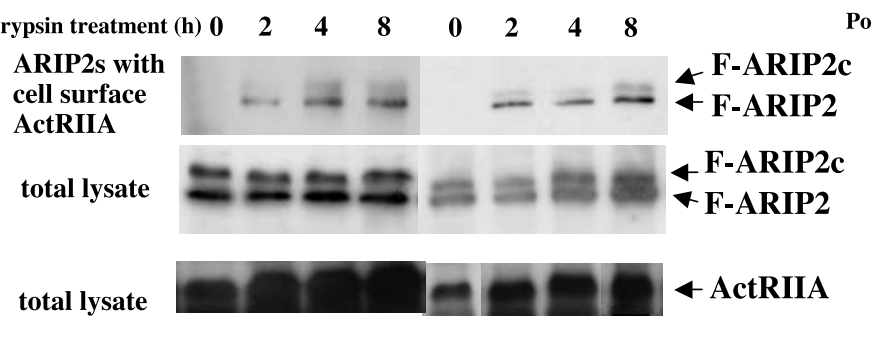

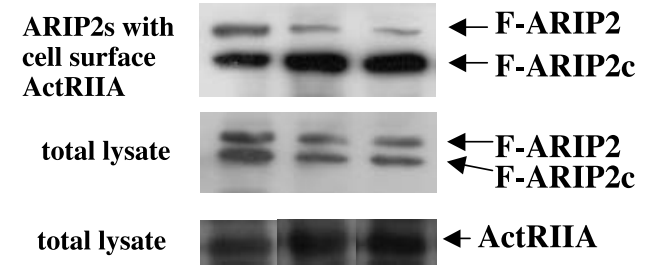

IP:myc WB:FLAG

Figure 7 ARIP2 is involved in endocytosis of ActRIIA, whereas ARIP2c regulates surface expression of ActRIIA. (A) HEK 293 cells were transfected with myc-tagged ActRIIA, FLAG (F)-ARIP2 and F-ARIP2c cDNAs. After $24 \mathrm{~h}$, cells were treated with trypsin, washed and then reincubated for appropriate time periods. Then, cell surface receptors were labeled with myc antibody, extracted, immunoprecipitated (IP) and blotted with FLAG antibody. In the middle and bottom rows, total lysate was analyzed. (B) Same experiment as in (A) except that cells were incubated in the presence of cycloheximide (CHX) for appropriate time periods after trypsin treatment to inhibit protein synthesis. (C) Same experiment as in (A) except that cells were incubated in the presence of chloroquine (left panel) or monensin (right panel) for appropriate time periods after trypsin treatment to inhibit recycling. (D) Same experiment as in left panel of (C) except that trypsin treatment was omitted. WB, Western blotting.

two isoforms of ARIP2, named ARIP2b and ARIP2c. Interestingly, unlike ARIP2, overexpression of ARIP2b or $2 \mathrm{c}$ enhanced activin signaling. ARIP1 and ARIP2 also interacted with ActRIIs, but they inhibited activin signal transduction when overexpressed (Shoji et al. 2000, Matsuzaki et al. 2002). ARIP2b and ARIP2c are unique in that they associate with ActRIIA and enhance activin signaling. In a previous study, we reported characterization of a PDZ domain deletion mutant of ARIP2 called ARIP2 $\Delta C$. Interestingly, ARIP2 $\Delta C$, like ARIP2b and 2c, enhances activin signaling (Matsuzaki et al. 2002). We did not detect significant interaction between ARIP2b/2c and ActRIIB. Similarly, there was no significant interaction between ARIP $2 b / 2 c$ and either TGF $\beta$ RII or BMPRII (Fig. 2A). Analysis of interactions between ARIP2b/2c and ActRIIAs showed that ARIP2b/2c interact with the $\mathrm{COOH}$ terminus of ActRIIAs through the PDZ domain. The PDZ domains of ARIP2 and its isoforms are identical. The three $\mathrm{COOH}$ terminal amino acids of ActRIIA, consistent with the PDZ-binding motif, are critical for this interaction (Fig. 2). ARIP2 interacts with ActRIIA via its PDZ domain and with RalBP1 via its $\mathrm{COOH}$-terminus, resulting in both endocytosis of activin type II receptors and an attenuated activin-induced transcriptional response (Matsuzaki et al. 2002). ARIP2b and $2 \mathrm{c}$ did not interact with RalBP1. Overexpression of ARIP2 $\mathrm{b}$ or $2 \mathrm{c}$ augmented activin-induced transcription in HEK 293 cells (Fig. 4A), and increased activin-induced secretion of FSH from L $\beta$ T2 cells (Fig. 4C). Further characterization revealed that, unlike ARIP2, ARIP2b/2c did not affect internalization of ActRII and showed antagonistic activity to ARIP2. Whether ARIP2b or $2 \mathrm{c}$ increase signal transduction in physiological conditions remains to be determined. Since ARIP2 and ARIP2b/2c have opposite effects on receptor localization and function, the ratio of ARIP2 to its isoforms, ARIP2b/2c, likely regulates ActRIIA activity. Our characterization of ARIP2 and its isoforms offers another example of isoforms having different functions.

Involvement of Smad proteins in cellular signaling induced by the TGF- $\beta$ superfamily has been extensively studied (Heldin et al. 1997). In addition, there is accumulating evidence that multiple adaptors and scaffolding proteins interact with receptor serine/threonine kinases. 
ARIP1 and ARIP2 interact with ActRIIA and ActRIIB. Dok-1 shows homologies to pleckstrin and to phosphotyrosine-binding domains that associate with activin and TGF- $\beta$ receptors, and is a key element in activin-mediated apoptosis in B cells (Yamakawa et al. 2002). TRIP1 is a WD domain-containing protein that interacts with TGF- $\beta$ type II receptors (Chen et al. 1995). TRAP-1-like protein (TLP) also associates with activin and TGF- $\beta$ receptors (Felici et al. 2003). Interestingly, TLP suppresses Smad3-dependent signaling but potentiates Smad2-dependent signaling. These results indicate that adaptor proteins have a role in coordinating signal transduction by regulating protein complexes including receptor serine/threonine kinase receptors. ActRIIA is shared with activins, myostatin, nodal and BMP-7. These growth factors are known to form ligand gradients. One potential mechanism for forming morphogenetic gradients involves regulation of receptor numbers at the cell surface (McDowell \& Gurdon 1999). Thus, ARIP2 and its variants may have a role in shaping morphogenetic gradients and in fine-tuning activin signaling during tissue formation (McDowell \& Gurdon 1999).

Our characterization of ARIP2 and its isoforms as mediators of activin signaling has revealed a novel regulation of receptor serine/threonine kinases, and adds new insights into the mechanisms of regulation of signal transduction through ActRII by PDZ proteins.

\section{Acknowledgements}

We thank Dr P Mellon (University of California, San Diego, CA, USA) for the kind gift of L $\beta$ T2 cells. The nucleotide sequence reported in this paper has been submitted to the GenBank/EMBL Data Bank with accession number AY071903 (ARIP2b). ARIP2c sequences are available under GenBank/EMBL accession number AK008254 by RIKEN.

\section{Funding}

This research was supported by the Ministry of Education, Culture, Sports, Science and Technology of Japan, and also by a Research Grant (16B-2) for Nervous and Mental Disorders, a Research Grant for Research on Psychiatric and Neurological Diseases and Mental Health from the Ministry of Health, Labour and Welfare (17231401), and by grants from the Sankyo Foundation of Life Science and Suzuken Memorial Foundation. The authors declare that there is no conflict of interest that would prejudice the impartiality of this scientific work.

\section{References}

Attisano L, Wrana JL, Cheifetz S \& Massagüe J 1992 Novel activin receptors: distinct genes and alternative mRNA splicing generate a repertoire of serine/threonine kinase receptors. Cell 68 97-108.
Attisano L, Wrana JL, Montalvo E \& Massagüe J 1996 Activation of signaling by the activin receptor complex. Molecular and Cellular Biology 16 1066-1073.

Bao YL, Tsuchida K, Liu B, Kurisaki A, Matsuzaki T \& Sugino H 2005 Synergistic activity of activin A and basic fibroblast growth factor on tyrosine hydroxylase expression through ERK1/ERK2 MAPK signaling pathways. Journal of Endocrinology 184 493-504.

Chen RH, Miettinen PJ, Maruoka EM, Choy L \& Derynck R 1995 A WW domain protein that is associated with and phosphorylated by the type II TGF-beta receptor. Nature 377 548-552.

Dennler S, Itoh S, Vivien D, ten Dijke P, Huet S \& Gauthier JM 1998 Direct binding of Smad3 and Smad4 to critical TGF $\beta$-inducible elements in the promoter of human plasminogen activator inhibitor-type 1 gene. EMBO Journal 17 3091-3100.

DiGuglielmo GM, Le Roy C, Goodfellow AF \& Wrana JL 2003 Distinct endocytic pathways regulate TGF-beta receptor signalling and turnover. Nature Cell Biology 5 410-421.

Ethier JF, Lussier JG \& Silversides DW 1997 Bovine activin receptor type IIB messenger ribonucleic acid displays alternative splicing involving a sequence homologous to src-homology 3 domain binding sites. Endocrinology 138 2425-2434.

Fanning AS \& Anderson JM 1999 Protein modules as organizers of membrane structure. Current Opinion in Cell Biology 11 432-439.

Felici A, Wurthner JU, Parks WT, Giam LR, Reiss M, Karpova TS, McNally JG \& Roberts AB 2003 TLP, a novel modulator of TGF signaling, has opposite effects on Smad2- and Smad3-dependent signaling. EMBO Journal 22 4465-4477.

Graham KE, Nusser KD \& Low MJ 1999 LbetaT2 gonadotroph cells secrete follicle stimulating hormone (FSH) in response to activin A. Journal of Endocrinology 162 R1-R5.

Heldin CH, Miyazono K \& ten Dijke P 1997 TGF-beta signalling from cell membrane to nucleus through SMAD proteins. Nature $390465-471$

Hirao K, Hata Y, Ide N, Takeuchi M, Irie M, Yao I, Deguchi M, Toyoda A, Sudhof TC \& Takai Y 1998 A novel multiple PDZ domain-containing molecule interacting with $\mathrm{N}$-methyl-D-aspartate receptors and neuronal cell adhesion proteins. Journal of Biological Chemistry 273 21105-21110.

McDowell N \& Gurdon JB 1999 Activin as a morphogen in Xenopus mesoderm induction. Seminars in Cell Development and Biology $10311-317$.

Mathews LS 1994 Activin receptors and cellular signaling by the receptor serine kinase family. Endocrine Reviews 15 310-325.

Mathews LS \& Vale WW 1991 Expression cloning of an activin receptor, a predicted transmembrane serine kinase. Cell 65 973-982.

Matsuzaki T, Hanai S, Kishi H, Liu ZH, Bao Y, Kikuchi A, Tsuchida K \& Sugino H 2002 Regulation of endocytosis of activin type II receptors by a novel PDZ protein through Ral/Ral-binding protein 1-dependent pathway. Journal of Biological Chemistry 277 19008-19018.

Nemoto Y \& DeCamilli P 1999 Recruitment of an alternatively spliced form of synaptojanin 2 to mitochondria by the interaction with the PDZ domain of a mitochondrial outer membrane protein. EMBO Journal 18 2991-3006.

Shoji H, Nakamura T, van den Eijnden-van Raaij AJ \& Sugino H 1998 Identification of a novel type II activin receptor, type IIA-N, induced during the neural differentiation of murine P19 embryonal carcinoma cells. Biochemical and Biophysical Research Communications 246 320-324.

Shoji H, Tsuchida K, Kishi H, Yamakawa N, Matsuzaki T, Liu ZH, Nakamura T \& Sugino H 2000 Identification and characterization of a PDZ protein that interacts with activin type II receptors. Journal of Biological Chemistry 275 5485-5492.

Songyang Z, Fanning AS, Fu C, Xu J, Marfatia SM, Chishti AH, Crompton AA, Chan AC, Anderson JM \& Cantley LC 1997 Recognition of unique carboxyl-terminal motifs by distinct PDZ domains. Science 275 73-77. 
Sugino H \& Tsuchida K 2000 Activin and Follistatin in Skeletal Growth Factors, pp 251-263. Ed E Canalis, Philadelphia, PA, USA:

Lippincott Williams \& Wilkins.

Tsuchida K 2004 Activins, myostatin and related TGF- $\beta$ family members as novel therapeutic targets for endocrine, metabolic and immune disorders. Current Drug Targets - Immune, Endocrine and Metabolic Disorders 4 157-166.

Tsuchida K, Vaughan JM, Wiater E, Gaddy-Kurtens D \& Vale WW 1995 Inactivation of activin-dependent transcription by kinase-deficient activin receptors. Endocrinology 136 5493-5503.
Tsuchida K, Matsuzaki T, Yamakawa N, Liu Z H \& Sugino H 2001 Intracellular and extracellular control of activin function by novel regulatory molecules. Molecular Cellular Endocrinology $18025-31$.

Yamakawa N, Tsuchida K \& Sugino H 2002 The rasGAP-binding protein, Dok-1, mediates activin signaling via serine/threonine kinase receptors. EMBO Journal 21 1684-1694.

Received in final form 1 February 2006

Accepted 9 February 2006 